\title{
DEFINABLE ENCODINGS IN THE COMPUTABLY ENUMERABLE SETS
}

PETER A. CHOLAK AND LEO A. HARRINGTON

\section{INTRODUCTION}

The purpose of this communication is to announce some recent results on the computably enumerable sets. There are two disjoint sets of results; the first involves invariant classes and the second involves automorphisms of the computably enumerable sets. What these results have in common is that the guts of the proofs of these theorems uses a new form of definable coding for the computably enumerable sets.

We will work in the structure of the computably enumerable sets. The language is just inclusion, $\subseteq$. This structure is called $\mathscr{E}$.

All sets will be computably enumerable non-computable sets and all degrees will computably enumerable and non-computable, unless otherwise noted. Our notation and definitions are standard and follow Soare [1987]; however we will warm up with some definitions and notation issues so the reader need not consult Soare [1987]. Some historical remarks follow in Section 2.1 and throughout Section 3.

1.1. Some definitions. We will also consider the quotient structure $\&$ modulo the ideal of finite sets, $\mathcal{E}^{*}$. $\mathcal{E}^{*}$ is a definable quotient structure of $\mathscr{E}$ since " $X$ is finite" is definable in $\varepsilon$; " $X$ is finite" iff all subsets of $X$ are computable (it takes a little computability theory to show if $X$ is infinite then $X$ has an infinite non-computable subset). We use $A^{*}$ to denote the equivalent class of $A$ under the ideal of finite sets. If there is an automorphism, $\Phi^{*}$, of $\varepsilon^{*}$ such that $\Phi^{*}\left(A^{*}\right)=B^{*}$ then there is an automorphism, $\Phi$, of $\&$ such that $\Phi(A)=B$ [see Soare, 1987, XV.2.7].

$\mathcal{L}(A)=\{W: A \subseteq W\}$ and $\mathcal{L}^{*}(A)=\left\{W^{*}: A \subseteq W\right\}$ is another pair of important definable substructures. If there is an automorphism $\Phi$ of $\mathscr{E}$ $\left(\Phi^{*}\right.$ of $\left.\mathcal{E}^{*}\right)$ such that $\Phi(A)=B\left(\Phi^{*}\left(A^{*}\right)=B^{*}\right)$ (in either case we say $A$ and $B$ are automorphic and write this as $A \simeq B$ ) then $\mathcal{L}^{*}(A)$ and $\mathcal{L}^{*}(B)$ are isomorphic structures (we write this as $\mathcal{L}^{*}(A) \simeq \mathcal{L}^{*}(B)$ ). A set $A$ is hyperhypersimple (written hhsimple) if $\mathcal{L}^{*}(A)$ is a Boolean algebra. A set

Research partially supported NSF Grants DMS-96-34565 (Cholak), DMS-96-22290 (Harrington). 
$M$ is maximal if $\mathcal{L}^{*}(A)$ is the two element Boolean algebra. A set $A$ is atomless if $A$ has no maximal superset.

We will need a way to classify the complexity of automorphisms of $\mathcal{E}$. Let $\Phi$ be an automorphism of $\mathcal{E}$. We say $\Phi$ is a $\Delta_{n}^{0}$-automorphism if there is a $\Delta_{n}^{0}$ function $f$ such that $\Phi\left(W_{e}\right)={ }^{*} W_{f(n)} . f$ is called a presentation of $\Phi$. Two sets $A$ and $B$ are $\Delta_{3}^{0}$-automorphic iff there is a $\Delta_{3}^{0}$-automorphism $\Phi$ such that $\Phi(A)=B$. This method of classication can also be used for isomorphisms between $\mathcal{L}^{*}(A)$ and $\mathcal{L}^{*}(B) . \mathcal{L}^{*}(A)$ and $\mathcal{L}^{*}(B)$ are $\Delta_{3}^{0}$ isomorphic (written $\mathcal{L}^{*}(A) \simeq{ }_{\Delta_{3}^{0}} \mathcal{L}^{*}(B)$ ) iff there is an isomorphism $\Phi^{*}$ between $\mathcal{L}^{*}(A)$ and $\mathcal{L}^{*}(B)$ and a $\Delta_{3}^{0}$ function $f$ such that $\Phi^{*}\left(W_{e}^{*}\right)=W_{f(e)}^{*}$.

We say a class $\mathscr{D}$ of degrees is invariant if there is a class $\&$ of computably enumerable sets such that if $\mathbf{d} \in \mathcal{D}$ then there is a $W$ in $\&$ and $\mathbf{d}$, if $W \in \&$ then $\operatorname{deg}(W) \in \mathscr{D}$ and $\&$ is closed under automorphisms of $\mathcal{E}$.

1.2. Invariant classes. The culmination of our work on invariant jump classes is the following:

Theorem 1.1. For all $n \geq 2$, the high ${ }_{n}\left(\overline{l_{\text {ow }}}\right)$ computably enumerable degrees are invariant.

As we will see later, this result resolves the long standing Martin's Invariance Conjecture (see Conjecture 2.2); the conjecture is not true. In Section 2, we will discuss our results concerning invariant degrees classes. Our results on invariant degrees classes will appear in Cholak and Harrington [n.d.b].

1.3. Automorphisms. Our work on automorphisms is slightly more technical and we will need a few definitions before we can state the main result.

Let $\delta(A)=\{B: \exists C(B \sqcup C=A)\} . \quad(A)$ is the splits of $A$ and $\&(A)$ forms a Boolean algebra. $\mathcal{R}(A)=\{R: R \subseteq A$ and $R$ is computable $\}$. $\mathcal{R}(A)$ is the computable subsets of $A$ and is an ideal of $\delta(A) . \wp_{\mathcal{R}}(A)$ is the quotient structure $\delta(A)$ modulo $\mathcal{R}(A)$. If $W \in S(A)$ then let $W^{R(A)}$ be the equivalent class of $W$ in $\varsigma_{\mathcal{R}}(A) . \varsigma_{\mathcal{R}}(A)$ is a Boolean algebra and is definable with a parameter for $A$. If $A$ and $B$ are automorphic then the structures $\delta_{\mathcal{R}}(A)$ and $\delta_{\mathcal{R}}(B)$ are isomorphic structures. But something much stronger is true.

Theorem 1.2. If $A$ and $B$ are automorphic then the structures $\varsigma_{\mathcal{R}}(A)$ and $\varsigma_{\mathcal{R}}(B)$ are $\Delta_{3}^{0}$-isomorphic structures (that is there is an isomorphism $\Psi$ between $\S_{\mathcal{R}}(A)$ and $\S_{\mathcal{R}}(B)$ and a $\Delta_{3}^{0}$-function $f$ such that for $W_{e} \in \&(A)$, $W_{f(e)}$ is in $\Psi\left(W^{\mathcal{R}(A)}\right)$ and we will write this as $\left.\delta_{\mathcal{R}}(A) \simeq_{\Delta_{3}^{0}} \delta_{\mathcal{R}}(B)\right)$. 
We will delay discussing the full impact and potential of the above theorem till Section 3. However we will mention one easy to understand theorem which follows from the above theorem and some work in Maass [1984]:

Theorem 1.3. Let $H_{1}$ and $H_{2}$ be hhsimple. $H_{1}$ and $H_{2}$ are automorphic iff they are $\Delta_{3}^{0}$-automorphic iff $\mathcal{L}^{*}\left(H_{1}\right) \simeq_{\Delta_{3}^{0}} \mathcal{L}^{*}\left(H_{2}\right)$.

What is exciting about this result is that it completely characterizes when two hhsimple sets are automorphic. They are automorphic iff their $\mathcal{L}^{*}$ 's are $\Delta_{3}^{0}$-isomorphic. We can easily work with the $\mathcal{L}^{*}$ 's as they are just Boolean algebras. In Section 3, we will mention how this theorem easily implies some known results. The results on automorphisms will appear in Cholak and Harrington [n.d.a].

1.4. Definable coding. As we mentioned earlier, the tie between these two disjoint sets of results is that their proofs use a new definable coding. The actual details of this coding is slightly complex. These details and some theorems concerning this coding will appear in Cholak and Harrington [n.d.b]. In what follows we will provide enough detail to understand our two applications.

The coding relies on the existence of infinitely many of what we call special $\mathcal{L}$-patterns $\mathcal{P}_{i}$ and formulas $\varphi_{\mathcal{P}}(A, \vec{U}, \vec{B}, S$ ) (where $\mathcal{L}=\{0,1,2,3\}$, $\vec{U}$ is a 4-tuple and $\vec{B}$ is an $n$-tuple for some $n$ determined by $\mathcal{P})$. What is important about $\varphi_{\mathcal{P}}(A, \vec{U}, \vec{B}, C)$ is that it is a statement in the language of $\{\subseteq\}$ (in fact it is an $\mathcal{L}(A)$-property), it is $\Sigma_{3}^{0}$ and it is invariant under automorphisms of $\mathcal{E}$ (so if $\Phi$ is an automorphism of $\mathscr{E}$ then $\varphi_{\mathcal{P}}(A, \vec{U}, \vec{B}, C$ ) iff $\left.\varphi_{\mathcal{P}}(\Phi(A), \Phi(\vec{U}), \Phi(\vec{B}), \Phi(C))\right)$. Informally in Section 4, we will describe the patterns $\mathcal{P}_{i}$ and discuss what $\varphi_{\mathcal{P}_{0}}(A, \vec{U}, \vec{B}, C)$ says.

Fix $\vec{U}$. Informally, we say $A$ realizes $\mathcal{P}_{i}(A$ might be $\emptyset)$ if for all $\vec{B}$ there is a $C$ (satisfying certain unnamed definable conditions) such that $\varphi_{\mathcal{P}_{i}}(A, \vec{U}, \vec{B}, C)$. The conditions on $C$ vary depending on the application of the coding. In the upcoming sections, we will briefly discuss the needed conditions for each application. In both cases, there is, uniformly, a universal $\vec{B}$; i.e. a $\vec{B}_{e}$ such that if $\varphi_{\mathcal{P}_{i}}\left(A, \vec{U}, \vec{B}_{e}, C\right)$ holds for some $C$ then $A$ realizes $\mathcal{P}_{i}$ (where $e$ codes $A, \vec{U}$ and $\mathcal{P}_{i}$ ).

The key idea is that it possible to construct sets (mainly $\vec{U}$ and $C$ ) to realize certain chosen $\mathcal{P}_{i}$ 's while avoiding the realization of the other $\mathcal{P}_{j}$ 's. This idea is at the technical heart of our results. In Cholak and Harrington [n.d.b, Section 3] we isolate this idea into a theorem. Also in Cholak and Harrington [n.d.b, Section 3] we refine the above notion of a universal $\vec{B}$ and prove a theorem that implies that universal $\vec{B}$ exist for each of our applications. 
We feel that this coding has great potential. In this paper, we have only mentioned our two recent applications. But surely there will be others. For example, one potential application of the coding is to use it to show that the $\exists \forall \exists$ theory of $\&^{*}$ is undecidable.

We were not the first to develop methods for coding in $\&$. Harrington and Herrmann developed various coding methods in $\mathcal{E}$. They both were able to use their methods to show that the theory of $\mathscr{E}$ is undecidable [Herrmann, 1984; Harrington, 1983]. Harrington was able to extend his methods to show that $\&$ has degree $\mathbf{0}^{(\omega)}$ [see Cholak, 1994]. The coding method used for this result has been further developed in Harrington and Nies [1998]. The culmination of these earlier methods of coding in $\varepsilon$ is Harrington's Ideal Definability Theorem which says that every $\Sigma_{k}^{0}$-ideal of $\varsigma_{\mathcal{R}}(A)$ is uniformly (in $k$ ) definable, where $k$ is odd and greater than 1 . The fact that the theorem is uniform allows us to quantify over the $\Sigma_{k}^{0}$-ideals for any $k$. Informally, to use this theorem one constructs a set $A$ and various definable ideals and then quantifying over the $\Sigma_{k}^{0}$-ideals allows us to encode the desired structure.

Our current coding is simpler to decode than the previous coding. More or less, it can be decoded at the $\Sigma_{3}^{A}$ level. Hence we should be able to use it to get sharper results. Our current coding follows naturally (to those familiar with automorphisms of $\mathbb{E}$ ) from how balls move and flow in the construction of automorphisms of $\mathscr{E}$ (see Section 4).

\section{Invariant Degree Classes}

2.1. History. Martin [1966] showed that the degrees of maximal sets are exactly the high degrees. Lachlan [1968a] and Shoenfield [1976] showed that the degrees of atomless sets are exactly the nonlow 2 degrees. So the high degrees and the nonlow 2 degrees are invariant. In addition, $\mathbf{L}_{\mathbf{0}}=\mathbf{0}$ and $\overline{\mathbf{L}_{\mathbf{0}}}=\mathbf{R}-\mathbf{0}$ are invariant as witnessed by the computable sets and non-computable sets, respectively. These results and Martin's work on projective determinacy led Martin to conjecture in the late 1960's:

Conjecture 2.1 (Martin's Original Invariance Conjecture). Among all nontrivial degree classes the invariant classes are exactly $\mathbf{H}_{\mathbf{2 n}-\mathbf{1}}$ and $\overline{\mathbf{L}_{2 \mathbf{n}}}$.

The alternation of every odd $\mathbf{H}_{\mathbf{n}}$ and even $\mathbf{L}_{\mathbf{n}}$ was inspired by the above mentioned results of Martin and Shoenfield and the behavior of projective determinacy. As more was known the conjecture was modified slightly. Lerman and Soare [1980] showed that the $d$-simple sets form a definable class which splits the low degrees. So at this time the conjecture was restricted to the jump classes $\mathbf{H}_{\mathbf{n}}$ and $\mathbf{L}_{\mathbf{n}}$, for $n>0$, and their complements. 
Conjecture 2.2 (Martin's Invariance Conjecture). Among the jump classes $\mathbf{H}_{\mathbf{n}}$ and $\mathbf{L}_{\mathbf{n}}$, for $n>0$, and their complements the invariant classes are exactly $\mathbf{H}_{\mathbf{2 n}-\mathbf{1}}$ and $\overline{\mathbf{L}_{\mathbf{2 n}}}$.

The conjecture was never published; the only published version can be found in Harrington and Soare [1996a]. Corollary 2.7 resolves the conjecture; it is not true. But first we will mention other related work.

Harrington in unpublished work showed that the property of being creative is elementary definable in $\mathcal{E}$ [see Soare, 1987, XV.1.1]. Hence $\mathbf{H}_{\mathbf{0}}$ is invariant. Harrington and Soare [1996b] showed that every prompt set is automorphic to a complete set and hence $\overline{\mathbf{H}_{0}}$ is non-invariant. Maass, Shore and Stob [1981] showed that there is a definable class of sets (the promptly simple sets - see below) which splits all jump classes. Also, in as yet unpublished work, Harrington and Soare [see Harrington and Soare, 1996a, Corollary 4.4] have shown that $\overline{\mathbf{L}_{1}}$ is not invariant further suggesting the Martin's conjecture is correct. They prove this by showing there is a properly low 2 degree $\mathbf{d}$ such that if $A \leq_{T} \mathbf{d}$ then there is a low $B$ such that $A$ and $B$ are automorphic. (Note the similarities between this and Conjecture 2.10.)

The conjecture divides into two types of jump classes: the upward closed jump classes, $\mathbf{H}_{\mathbf{n}}$ and $\overline{\mathbf{L}_{\mathbf{n}}}$, for all $n$, and the downward closed jump classes, $\mathbf{L}_{\mathbf{n}}$ and $\overline{\mathbf{H}_{\mathbf{n}}}$, for all $n$. The conjecture implies that no downward closed jump classes are invariant. This was verified earlier. Cholak [1995] and, independently, Harrington and Soare [1996b] showed that every non-computable set is automorphic to a high set. So the only invariant jump classes must be upward closed.

Downey and Harrington [1996] have shown that there is no fat orbit. That is they showed there is a definable property $S(A)$, a prompt $\mathbf{L}_{\mathbf{1}}$ degree $\mathbf{d}_{\mathbf{1}}$, a prompt $\mathbf{H}_{\mathbf{2}}$ degree $\mathbf{d}_{\mathbf{2}}$ greater than $\mathbf{d}_{\mathbf{1}}$, and tardy (non-prompt) $\mathbf{H}_{\mathbf{2}}$ degree $\mathbf{e}$ such that for all $E \leq_{T} \mathbf{e}, \neg S(E)$ and if $\mathbf{d}_{\mathbf{1}} \leq_{T} D \leq_{T} \mathbf{d}_{\mathbf{2}}$ then $S(D)$. This result points out the dichotomy between the prompt and tardy degrees. A corollary is, except for the high degrees, no single orbit can witness that a member of the $\mathbf{H}_{n}, \mathbf{L}_{n}$ hierarchy is invariant.

It is known that the prompt degrees are invariant: Maass [1983] showed that all promptly simple sets with semilow complements are automorphic. Maass et al. [1981] show that every promptly simple set has the splitting property (a definable property see Maass et al. [1981] for details) and every set which has the splitting property is prompt. Now it enough to show that there is a promptly simple set with semilow complement in every prompt degree. One such proof can be found in Wald [1999, Theorem 1.4.1].

2.2. Our work. We say $X$ is computable modulo $A$ iff there is a $Y$ such that $X \cap Y \subseteq A$ and $X \cup Y \cup A=\omega$ or, equivalently, there is a computable $R$ 
such that $R \subseteq X$ and $X \subseteq R \cup A$. We need to formalize when $A$ realizes $\mathcal{P}_{i}$. In Cholak and Harrington [n.d.b], we define the set $g_{A, \vec{U}}$. So for a fixed $\vec{U}$, $A$ realizes $\mathcal{P}$ iff $\mathcal{P} \in \mathcal{g}_{A, \vec{U}}$. Here we will not provide the actual definition of $\mathcal{P} \in \mathscr{g}_{A, \vec{U}}$ but rather an equivalent statement. Proving these two statements are equivalent is a simple application of a theorem about special $\mathcal{L}$-patterns which says that in this situation universal special $\mathcal{L}$-patterns exists. $\vec{B}_{e}$ is the universal special $\mathcal{L}$-pattern which can be found uniformly from any $e$ coding $A, \vec{U}$, and $\mathcal{P}$.

Lemma 2.3. $\mathscr{F}_{A, \vec{U}}$ is the set of special $\mathcal{L}$-patterns, $\mathcal{P}$, such that there exists $e$, there is a split $S$ of $W_{e}=C$ such that $S$ is not computable modulo $A$ and $\varphi_{\mathcal{P}}\left(A, \vec{U}, \vec{B}_{e}, S\right)$.

Now by counting quantifiers one can see that $g_{A, \vec{U}}$ is $\Sigma_{4}^{0}$. But we can improve this to show that $g_{A, \vec{U}}$ is $\Sigma_{3}^{A}$. This requires a theorem and breaks into two cases: when $A$ is high and otherwise. Now we are in a position to state our main theorem on invariant classes.

Theorem 2.4. There are special $\mathcal{L}$-patterns $\mathcal{P}_{i}$ 's $\left(\mathcal{P}_{0}\right.$ is informally described in Section 4) such that for all computably enumerable $D$ and all sets $J$, if $J$ is $\Sigma_{3}^{D}$ then there is a computably enumerable $A$ and there is a $\mathcal{L}$-interpretation $\vec{U}$ such that $A \equiv_{T} D$ and $i \in J$ iff $\mathcal{P}_{i} \in \mathcal{g}_{A, \vec{U}}$.

Definition 2.5. Let $\varphi_{J}(A)$ be the $\mathscr{L}_{\omega, \omega_{1}}$ sentence: There exists an $\mathcal{L}$ interpretation $\vec{U}$, for all $i, i \in J$ iff $\mathcal{P}_{i} \in \mathcal{g}_{A, \vec{U}}$.

$\varphi_{J}(A)$ is a property of $\mathcal{L}(A)$. If $A$ and $B$ are automorphic then $\varphi_{J}(A)$ iff $\varphi_{J}(B)$. In addition, we can show that if $\varphi_{J}(A)$ then $J$ is $\Sigma_{3}^{A}$. In the proof of Theorem 2.4, we employ our "key idea"; we can selectively realize different $\mathcal{P}_{i}$ 's. We can use Theorem 2.4 to encode information, particularly about the double jump.

Theorem 2.6. Let $\mathcal{C}=\left\{\mathbf{a}: \mathbf{a}\right.$ is the Turing degree of a $\Sigma_{3}^{0}$ set $\left.J \geq_{T} \mathbf{0}^{\prime \prime}\right\}$. Let $\mathcal{D} \subseteq \mathcal{C}$ be such that $\mathcal{D}$ is upward closed. Then there is an $\mathcal{L}(A)$ property $\varphi_{\mathscr{D}}(A)$ such that $D^{\prime \prime} \in \mathcal{D}$ iff there is an $A$ such that $A \equiv D$ and $\varphi_{D}(A)$.

Proof. Let $\varphi_{\mathscr{D}}(A)$ be the infinite disjunction of all $\varphi_{J}(A)$ where $J$ is a $\Pi_{3}^{0}$ set whose Turing degree is in $\mathscr{D}$. If $D^{\prime \prime} \in \mathscr{D}$ then there is a $\Pi_{3}^{0}$ set $J$ in the above disjunction such that $J$ is $\Sigma_{3}^{D}$ and hence there is an $A$ such that $A \equiv_{T} D$ and $\varphi_{J}(A)$. Assume there is an $A$ such that $A \equiv_{T} D$ and $\varphi_{J}(A)$, for some $J$ in the above disjunction. Then $J$ is $\Sigma_{3}^{A}$, and, since $J$ is $\Pi_{3}^{0}, J$ is $\Delta_{2}^{A}$ and $\Delta_{2}^{D}$. So $J \leq_{T} D^{\prime \prime}$ and $D^{\prime \prime} \in \mathscr{D}$. 
Corollary 2.7. For all $n \geq 2$, the high $h_{n}\left(\overline{\text { low }_{n}}\right)$ computably enumerable degrees are invariant.

Proof. Let $\mathcal{D}=\{\mathbf{a}: \mathbf{a}$ is the Turing degree of the double jump of some $\operatorname{high}_{n}\left(\overline{\operatorname{low}_{n}}\right)$ degree\}. So $\varphi_{\mathscr{D}}(A)$ iff $A$ is $\operatorname{high}_{n}\left(\overline{\operatorname{low}_{n}}\right)$.

Corollary 2.8. Let $\mathcal{F}$ be a class of computably enumerable degrees such that if $\mathbf{a} \in \mathcal{F}$ and $\mathbf{a}^{\prime \prime} \leq_{T} \mathbf{b}^{\prime \prime}$ then $\mathbf{b} \in \mathcal{F}$. Then $\mathcal{F}$ is invariant.

Proof. Let $\mathcal{D}=\left\{\mathbf{a}^{\prime \prime}: \mathbf{a} \in \mathcal{F}\right\}$.

Corollary 2.9. If $\mathbf{a}^{\prime \prime}>\mathbf{b}^{\prime \prime}$ then there is an $A \in \mathbf{a}$ such that for all $B \in \mathbf{b}$, $A \not \subset B$ (in fact, $\mathcal{L}(A)$ and $\mathcal{L}(B)$ are not isomorphic).

Proof. Let $\mathscr{D}=\left\{\mathbf{d}^{\prime \prime}: \mathbf{d} \geq_{T} \mathbf{a}\right\}$. Now there is a $A \in \mathbf{a}$ such that $\varphi_{\mathscr{D}}(A)$. If $B \in \mathbf{b}$ then $\neg \varphi_{\mathscr{D}}(B)$. So $A$ and $B$ cannot have isomorphic $\mathcal{L}$ 's.

2.3. Some open questions. More or less we have shown that the double jump can be encoded into $\mathcal{E}$. What about the single jump? We conjecture that this cannot be done. The following conjecture is the strongest possible negation of encoding the single jump in the above fashion (see Corollary 2.9) and is a generalization of the result of Harrington and Soare that $\overline{\mathbf{L}} \mathbf{1}$ is noninvariant.

Conjecture 2.10. Let $J$ be c.e. in and above $\mathbf{0}^{\prime \prime}$. There are degrees $\mathbf{a}$ and $\mathbf{b}$ such that $\mathbf{a}^{\prime} \neq \mathbf{b}^{\prime}, \mathbf{a}^{\prime \prime} \equiv_{T} \mathbf{b}^{\prime \prime} \equiv_{T} J$, and for all $A \leq_{T}$ a there is a $B$ such that $B \leq_{T} \mathbf{b}$ and $A \simeq B$.

The no fat orbit result of Downey and Harrington [1996] says the degrees in the above conjecture mostly likely are both prompt or tardy. So the fact that the prompt degrees are invariant provides some evidence the conjecture should be true. In addition, we have some partial results along this direction:

Theorem 2.11 (Cholak [1995]). For all $A$ and for all $\mathbf{H}_{\mathbf{1}}$ degrees $\mathbf{h}$ there is a $B \in \mathbf{h}$ such that $\mathcal{L}^{*}(A) \simeq \mathcal{L}^{*}(B)$.

Recently Harrington has announced a generalization of the above theorem:

Theorem 2.12 (Harrington). For all $A$ and degrees $\mathbf{d}$ if $A^{\prime} \leq_{T} \mathbf{d}^{\prime}$ there is a $B \in \mathbf{d}$ such that $\mathcal{L}^{*}(A) \simeq \mathcal{L}^{*}(B)$.

\section{Automorphisms}

Our understanding of automorphism of $\mathcal{E}$ is unique to $\mathcal{E}$. In most structures with non-trivial automorphisms we can construct automorphisms via the normal "back and forth" argument. But this is not the case with $\mathcal{E}$. To construct automorphisms we use the properties of being well-visited and 
well-resided. Well-visited is $\Pi_{2}^{0}$ and not being well-resided is $\Sigma_{3}^{0}$ (we use the negation). Since the complexity of these properties is at most $\Sigma_{3}^{0}$, the construction of the desired automorphism can be placed on a tree. (We will not discuss the details on this placement nor of the construction of an automorphism of $\&$ but direct the reader to Harrington and Soare [1996b] or Cholak [1995].)

If $\Phi$ is constructed on a tree then $\Phi$ has a presentation computable in the true path (which is $\Delta_{3}^{0}$ ). Hence all automorphism constructed in this way are $\Delta_{3}^{0}$-automorphisms (or even effective automorphisms).

Lachlan [see Soare, 1987, Theorem XV.2.2] showed that $\&$ has $2^{\aleph_{0}}$ automorphisms. Hence there must be automorphisms which are not $\Delta_{3}^{0}$. Even with Lachlan's result there was some slight hope that the relation of whether two sets are automorphic would be $\Delta_{3}^{0}$ or at least arithmetic. But the as yet unpublished proof of the Slaman-Woodin Conjecture shows this is not the case:

Theorem 3.1 (Cholak, Downey and Harrington [n.d.]). The set $\{(A, B): A \simeq B\}$ is $\Sigma_{1}^{1}$-complete.

Therefore we know that there are useful non- $\Delta_{3}^{0}$-automorphisms. But the proof of this theorem does not allow us to clearly lay our hands on any such automorphisms. The automorphisms constructed for this theorem are based on complex arrangements of $\Delta_{3}^{0}$-isomorphisms. (The automorphisms constructed in Lachlan's proof are complex arrangements of effective isomorphisms.) Thus there is some hope that all automorphisms are just complex arrangements of $\Delta_{3}^{0}$-isomorphisms.

Theorem 1.2 provides us with some factual basis for this hope. By Theorem 3.1 we cannot go from a $\Delta_{3}^{0}$ isomorphism from $\varsigma_{\mathcal{R}}(A)$ to $\varsigma_{\mathcal{R}}(B)$ to an automorphism of $\varepsilon$ taking $A$ to $B$. But there is the hope that we can add extra conditions to this $\Delta_{3}^{0}$ isomorphism to help construct the desired automorphism. Theorem 1.2 also provides evidence that we are limited to our current methods in how we can construct automorphisms of $\mathcal{E}$. This is certainly the case for hhsimple sets (see below).

Theorem 1.2 will also have an impact in showing that certain sets cannot be automorphic. Most such results go more or less as follows: First show that the sets $A$ and $B$ cannot be $\Delta_{3}^{0}$-automorphic. Then use the failure to be $\Delta_{3}^{0}$-automorphic to find a definable property $P$ true of $A$ but not of $B$. For example, in our quest to prove Theorems 2.4 and 2.6, we first showed that if $\mathbf{a}^{\prime \prime}>\mathbf{b}^{\prime \prime}$ then there is an $A \in \mathbf{a}$ such that for all $B \in \mathbf{b}, A \chi_{\Delta_{3}^{0}} B$ (compare this statement to Theorems 2.9 and Conjecture 2.10). By examining this proof we were able to come up with our coding and Theorems 2.4 and 2.6. By using Theorem 1.2 perhaps we can formalize this process. If not then at 
least we can use Theorem 1.2 to diagonalize against all $\Delta_{3}^{0}$-isomorphisms and show that certain sets are not automorphic without producing a definable difference.

3.1. Automorphisms and hhsimple sets. By Lachlan [1968b] [see Soare, 1987, Corollary X.7.2] a set $H$ is hhsimple iff $\mathcal{L}^{*}(A)$ is a $\Sigma_{3}^{0}$-Boolean algebra. We have a good understanding of computable Boolean algebras [see Remmel, 1989] and we can get most of what we need by relativizing these results. Hence hhsimple sets are easier to work with than a random computably enumerable set. For example, one of the key facts Soare used in his classical proof that the maximal sets are automorphic [Soare, 1974] [see Soare, 1987, XV.4.6] is that if $M$ is maximal then $\mathcal{L}^{*}(M)$ is the 2 element Boolean algebra. Maass [1984] extended Soare's work to show that if $H_{1}$ and $H_{2}$ are hhsimple sets and $\mathcal{L}^{*}\left(H_{1}\right) \simeq_{\Delta_{3}^{0}} \mathcal{L}^{*}\left(H_{2}\right)$ then $H_{1}$ and $H_{2}$ are $\Delta_{3}^{0}$-automorphic.

The work on hhsimple sets and automorphisms of $\&$ is aimed at answering the question of when are two random hhsimple sets with the isomorphic $\mathcal{L}^{*}$ automorphic. Theorem 1.3 provides the complete answer; hhsimple sets are automorphic iff their $\mathcal{L}^{*}$ 's are $\Delta_{3}^{0}$-isomorphic.

One known corollary of Theorem 1.3 is that there are hhsimple sets which are not automorphic but yet have isomorphic $\mathcal{L}^{*}$ 's: just build two isomorphic computable Boolean algebras $\mathscr{B}_{0}$ and $\mathscr{B}_{1}$ which are not $\Delta_{3}^{0}$-isomorphic and apply the above mentioned theorem of Lachlan to construct hhsimple sets such that $\mathcal{L}^{*}\left(H_{i}\right)=\mathscr{B}_{i}$. This result is due to Lerman, Shore and Soare [1978]. Lerman et al. [1978] construct a pair of hhsimple sets $H_{0}$ and $H_{1}$ such that $\mathcal{L}^{*}\left(H_{0}\right)$ and $\mathcal{L}^{*}\left(H_{1}\right)$ are isomorphic to the atomless Boolean algebra and $H_{0}$ has a $r$-maximal major subset (a definable property in $\mathcal{E}$ ) and $H_{1}$ does not have such a subset and hence $H_{0}$ and $H_{2}$ are not automorphic. This result was later extended in Herrmann [1989]. He showed that there is a definable structure $P_{I}^{*}(H)$ such that if $H_{1}$ and $H_{2}$ are automorphic then $P_{I}^{*}\left(H_{1}\right)$ and $P_{I}^{*}\left(H_{2}\right)$ are isomorphic and, for any possible $\mathcal{L}^{*}$, he constructed pairwise nonautomorphic hhsimple sets $H_{0}$ and $H_{1}$ such that $P_{I}^{*}\left(H_{1}\right)$ and $P_{I}^{*}\left(H_{2}\right)$ are not isomorphic but $\mathcal{L}^{*}\left(H_{0}\right)$ and $\mathcal{L}^{*}\left(H_{1}\right)$ are isomorphic to $\mathcal{L}^{*}$. Herrmann [1989] asked if having isomorphic $P_{I}^{*}$ s $\mathrm{s}$ is sufficient to show hhsimple sets are automorphic. We conjecture the question is now answerable: $\mathcal{L}^{*}\left(H_{0}\right) \simeq{ }_{\Delta_{3}^{0}} \mathcal{L}^{*}\left(H_{1}\right)$ implies either $P_{I}^{*}\left(H_{1}\right)$ and $P_{I}^{*}\left(H_{2}\right)$ are isomorphic or that they are not.

Theorem 1.3 also improves some unpublished work of Harrington. Harrington [see Cholak, 1994] showed that if $H_{1}$ and $H_{2}$ are automorphic 
hhsimple sets then their $\mathcal{L}^{*}$ 's are arithmetically isomorphic. In fact, $\Delta_{n}^{0}$ isomorphic for some $n$ between 7 and 9 but no one counted very carefully. This uses the coding methods described in Harrington and Nies [1998].

Another corollary of Theorem 1.3 (or the above mentioned theorem of Harrington) is that Slaman and Woodin's proof that the set $\left\{\left\langle e_{0}, e_{1}\right\rangle: \mathcal{L}^{*}\left(W_{e_{0}}\right)\right.$ isomorphic to $\left.\mathcal{L}^{*}\left(W_{e_{1}}\right)\right\}$ is $\Sigma_{1}^{1}$-complete cannot be improved to provide a proof of the SlamanWoodin Conjecture (see Theorem 3.1). The proof of Slaman and Woodin's result goes something like this: Build a uniformly computable collection of computable Boolean algebras $\left\{\mathscr{B}_{i}\right\}_{i \in \omega}$ such that the set $\left\{\langle i, j\rangle: \mathscr{B}_{i}\right.$ is isomorphic to $\left.\mathscr{B}_{j}\right\}$ is $\Sigma_{1}^{1}$-complete. Now to this computable collection apply Lachlan's [Lachlan, 1968b] construction of a hhsimple set to get a computable collection of computably enumerable sets $\left\{A_{i}\right\}_{i \in \omega}$ where $\mathcal{L}^{*}\left(A_{i}\right)$ is isomorphic to $\mathscr{B}_{i}$. So they actually show that $\left\{\left\langle e_{0}, e_{1}\right\rangle: \mathcal{L}^{*}\left(W_{e_{0}}\right)\right.$ isomorphic to $\mathcal{L}^{*}\left(W_{e_{1}}\right) \wedge W_{e_{0}}$ is hhsimple $\}$ is $\Sigma_{1}^{1}$-complete. Whether hhsimple sets are automorphic is an arithmetic question since it quantifies over $\Delta_{3}^{0}$-automorphisms.

3.2. A quick sketch of Theorem 1.2. Given $A$ and an automorphism $\Phi$ we will construct subsets of $A, B$ and $C$, with certain definable and dynamic properties (actually we build $\Phi(C)$ to have certain dynamic properties). Using a tree argument, we associate in a definable and $\Delta_{3}^{0}$ fashion the $i$ th non-computable split $T$ of $A$ with a split $S$ of $C$. $S$ will be constructed such that $S \cap T$ only realizes $\mathcal{P}_{2 i}$ and $S \cap \bar{T}$ only realizes $\mathcal{P}_{2 i+1}$. It is at this point in the proof that we use the fact that we can selectively realize different $\mathcal{P}_{i}$ 's. But to ensure that this actually does determine $T$ modulo $\mathcal{R}(A)$, we will ensure that the split $S$ will be constructed so that it is robust with respect to $A$ (namely, for all splits $U$ of $A$, if $U$ is not computable then $(U \cap S)$ is not computable). We also construct a $\Sigma_{3}^{0}$-filter $\mathcal{F}$ of robust splits of $C$; the split $S$ is constructed to be a split of some $D$ in $\mathcal{F}$, having a certain definable property $P$ with respect to $D, B$ and $A$.

We must decode this association. Let $\widehat{W}=\Phi(W)$. We must associate in a $\Sigma_{3}^{0}$ way a split $\tilde{T}$ of $\widehat{A}$ with a robust (with respect to $\widehat{A}$ ) split $\tilde{S}$ of $\widehat{C}$ such that they have the encoding property for some $i$. By Harrington's Ideal Definability Lemma, given $\mathcal{F}$ there is a corresponding $\Delta_{3}^{0}$-filter $\widehat{\mathcal{F}}$. So for some $\widehat{D} \in \widehat{\mathcal{F}}, \widehat{S}$ has the definable property $P$ with respect to $\widehat{D}, \widehat{B}$ and $\widehat{A}$. The definable property $P$ is not $\Sigma_{3}^{0}$, but, in the presence of certain unnamed dynamic properties of $\widehat{C}$, it implies a certain $\Sigma_{3}^{0}$ property of $\tilde{S}, \tilde{D}, \widehat{B}$ and $\widehat{A}$ which in turn ensures that $\tilde{S}$ inherits robustness from $\tilde{D}$. Hence in a $\Delta_{3}^{0}$ way we can associate $\tilde{T}$ with a robust split $\tilde{S}$ of $\widehat{C}$ so that they have the encoding property for some $i$.

We will map the $i$ th non-computable split $T$ of $A$ to a split $\tilde{T}$ of $\widehat{A}$ whose associated robust split $\tilde{S}$ of $\widehat{C}$ satisfies $\tilde{S} \cap \tilde{T}$ realizes $\mathcal{P}_{2 i}$ and $\tilde{S} \cap \overline{\tilde{T}}$ realizes 
$\mathcal{P}_{2 i+1}$. This mapping is $\Delta_{3}^{0}$. For more details we direct the reader to Cholak and Harrington [n.d.a].

3.3. A quick sketch of Theorem 1.3. First we need some fact about hhsimple sets. If $W$ is a superset of a hhsimple set $H$ then there is a computable set $R$ such that $W \cap \bar{H}=R \cap \bar{H}$. Hence each superset $W$ of $H$ gives rise to a splitting of $H ; R \cap H$ and $\bar{R} \cap H$. Unless $W \subseteq \subseteq^{*} H$ or $\bar{H} \subseteq *$ neither $R \cap H$ nor $\bar{R} \cap H$ are computable subsets of $H$.

We can make this relationship more formal: Fix a hhsimple set $H$. Let $\tilde{\mathcal{L}}(H)$ be the definable quotient substructure of $\delta_{\mathcal{R}}(H)$ given by $\{R \cap H: R$ is computable $\}$ modulo $\mathcal{R}(H)$. Let $H$ be hhsimple. Then it is easy to show that $\mathcal{L}^{*}(H)$ and $\tilde{\mathcal{L}}(H)$ are $\Delta_{3}^{0}$-isomorphic (for the details see Cholak and Harrington [n.d.a]).

This fact, Theorem 1.2 and the above mentioned theorem of Maass provide a proof of Theorem 1.3. The second "iff" follows directly from Maass's work. The "only if" direction of the first "iff" is clear. Assume $H_{1}$ and $H_{2}$ are automorphic hhsimple sets. Then, by Theorem 1.2, $\delta_{\mathcal{R}}\left(H_{1}\right) \simeq{ }_{\Delta_{3}^{0}} \delta_{\mathcal{R}}\left(H_{2}\right)$. This is enough to show $\tilde{\mathcal{L}}\left(H_{1}\right)$ and $\tilde{\mathcal{L}}\left(H_{2}\right)$ are $\Delta_{3}^{0}$-isomorphic and hence $\mathcal{L}\left(H_{1}\right)$ and $\mathcal{L}\left(H_{2}\right)$ are $\Delta_{3}^{0}$-isomorphic.

\section{THE SPECIAL $\mathcal{L}$-PATTERNS}

In this section we give an informal sketch about the special $\mathcal{L}$-patterns and how they are realized. Recall that informally, we say $A$ realizes $\mathcal{P}_{i}(A$ might be $\emptyset$ ) if for all $\vec{B}$ there is a $C$ (satisfying certain unnamed definable conditions) such that $\varphi_{\mathcal{P}_{i}}(A, \vec{U}, \vec{B}, C)$. We will ignore the particular application and the conditions the application imposes on $C$. Fix a $\vec{B}$. Informally we consider what it means for $\varphi_{\mathcal{P}_{0}}(A, \vec{U}, \vec{B}, C)$ to hold.

We think of the Figure 1 as a tree with some additional information. We think of balls $x$ in $C-A$ as moving down the tree. Each node $p$ is presented by a set $R_{p} . \varphi_{\mathcal{P}_{0}}(A, \vec{U}, \vec{B}, C)$ holds iff there is a $\vec{R}$ with the properties described below. A ball $x$ is at $p$ iff $x \in R_{q}$, for all $q \geq p$ (our trees go downwards). We will assume that every ball in $C-A$ is at some node. If $x$ is at a node labeled by $U_{i}$ then $x$ should also be in $U_{i}$. There are two ways to move downwards in the pattern; a BLUE move or a RED move. There is only one RED move in $\mathcal{P}_{0}$; at the node labeled by $\mathcal{R}\left(b_{2}\right)$. Every ball which enters $R_{b_{2}}$ must move downwards; i.e. must enter one of $R_{r_{1}}$ or $R_{r_{2}}$. All the nodes labeled with a $b_{i}$ have BLUE moves associated with them. A ball $x$ can only enter $R_{b_{i}}$ iff $x$ is at the node above $b_{i}$ and $x$ enters $B_{b_{i}}$.

For special $\mathcal{L}$-pattern $\mathcal{P}_{n}$ we just repeat the part of the pattern in the box in $\mathcal{P}_{0} n+1$ times. We call this part of the pattern a block. So balls can flow from a copy of $b_{4}$ to another copy of $b_{2}$. 


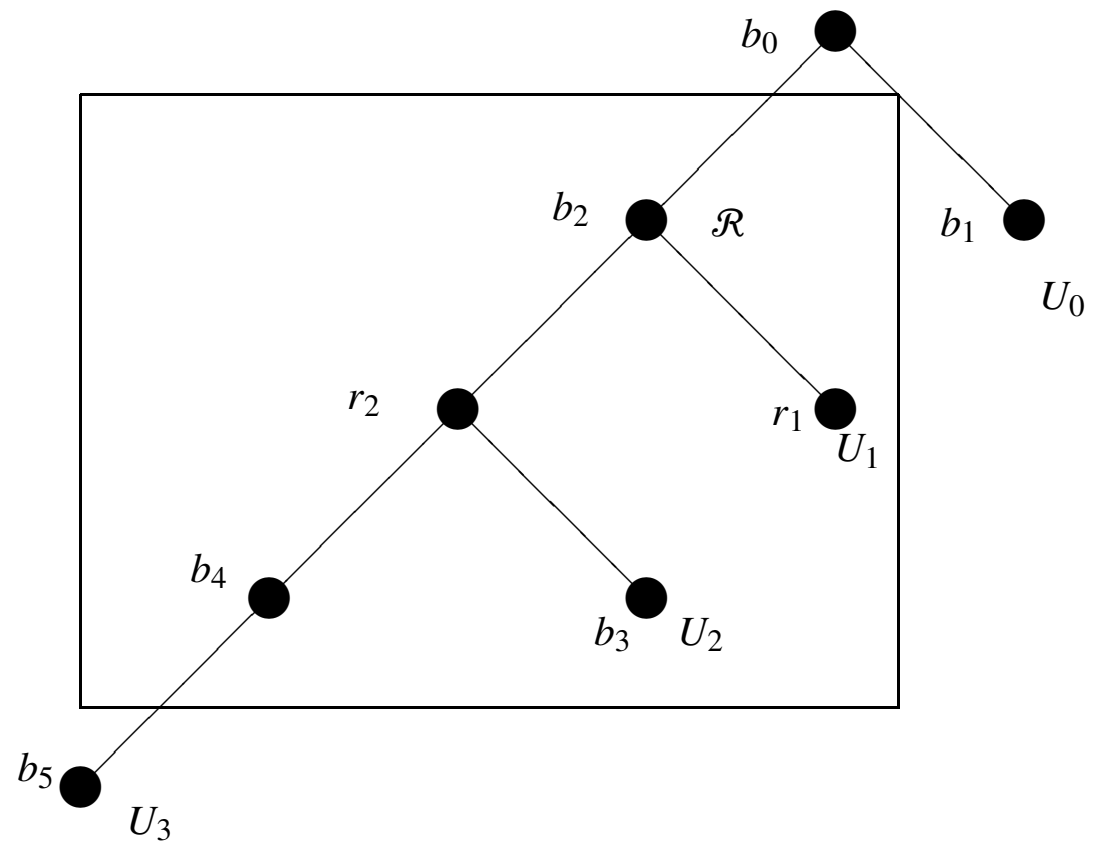

FIgURE I. The special $\mathcal{L}$-pattern $\mathcal{P}_{0}$.

\section{REFERENCES}

Cholak, P. [1994]. Notes on 3 theorems by Leo Harrington. Handwritten Notes. 1.4, 3.1

Cholak, P. [1995]. Automorphisms of the lattice of recursively enumerable sets, Mem. Amer. Math. Soc. 113(541): viii+151. 2.1, 2.11, 3

Cholak, P., Downey, R. and Harrington, L. A. [n.d.]. Automorphisms of the computably enumerable sets: $\Sigma_{1}^{1}$-completeness. In preparation. 3.1

Cholak, P. and Harrington, L. A. [n.d.a]. $\Delta_{3}^{0}$-automorphisms of the computably enumerable sets. In Preparation. 1.3, 3.2, 3.3

Cholak, P. and Harrington, L. A. [n.d.b]. Definable encodings in the computably enumerable sets. Submitted, Draft available. 1.2, 1.4, 2.2

Downey, R. and Harrington, L. [1996]. There is no fat orbit, Ann. Pure Appl. Logic 80(3): 277-289. 2.1, 2.3

Harrington, L. A. [1983]. The undecidability of the lattice of recursively enumerable sets. Handwritten Notes. 1.4

Harrington, L. A. and Nies, A. [1998]. Coding in the partial order of enumerable sets, $A d v$. Math. 133(1): 133-162. 1.4, 3.1

Harrington, L. A. and Soare, R. I. [1996a]. Definability, automorphisms, and dynamic properties of computably enumerable sets, Bull. Symbolic Logic 2(2): 199-213. 2.1 
Harrington, L. A. and Soare, R. I. [1996b]. The $\Delta_{3}^{0}$-automorphism method and noninvariant classes of degrees, J. Amer. Math. Soc. 9(3): 617-666. 2.1, 3

Herrmann, E. [1984]. The undecidability of the elementary theory of the lattice of recursively enumerable sets, Frege conference, 1984 (Schwerin, 1984), Akademie-Verlag, Berlin, pp. 66-72. 1.4

Herrmann, E. [1989]. Automorphisms of the lattice of recursively enumerable sets and hyperhypersimple sets, Logic, methodology and philosophy of science, VIII (Moscow, 1987), North-Holland, Amsterdam, pp. 179-190. 3.1

Lachlan, A. H. [1968a]. Degrees of recursively enumerable sets which have no maximal supersets., J. Symbolic Logic 33: 431-443. 2.1

Lachlan, A. H. [1968b]. On the lattice of recursively enumerable sets, Trans. Amer. Math. Soc. 130: 1-37. 3.1

Lerman, M., Shore, R. A. and Soare, R. I. [1978]. $r$-maximal major subsets, Israel J. Math. 31(1): 1-18. 3.1

Lerman, M. and Soare, R. I. [1980]. $d$-simple sets, small sets, and degree classes, Pacific J. Math. 87(1): 135-155. 2.1

Maass, W. [1983]. Characterization of recursively enumerable sets with supersets effectively isomorphic to all recursively enumerable sets, Trans. Amer. Math. Soc. 279: 311-336. 2.1

Maass, W. [1984]. On the orbit of hyperhypersimple sets, J. Symbolic Logic 49: 51-62. $1.3,3.1$

Maass, W., Shore, R. A. and Stob, M. [1981]. Splitting properties and jump classes, Israel J. Math. 39: 210-224. 2.1

Martin, D. A. [1966]. Classes of recursively enumerable sets and degrees of unsolvability, Z. Math. Logik Grundlag. Math. 12: 295-310. 2.1

Remmel, J. B. [1989]. Recursive Boolean algebras, Handbook of Boolean algebras, Vol. 3, North-Holland, Amsterdam, pp. 1097-1165. 3.1

Shoenfield, J. R. [1976]. Degrees of classes of recursively enumerable sets, J. Symbolic Logic 41: 695-696. 2.1

Soare, R. I. [1974]. Automorphisms of the lattice of recursively enumerable sets I: maximal sets, Ann. of Math. (2) 100: 80-120. 3.1

Soare, R. I. [1987]. Recursively Enumerable Sets and Degrees, Perspectives in Mathematical Logic, Omega Series, Springer-Verlag, Heidelberg. 1, 1.1, 2.1, 3, 3.1

Wald, K. [1999]. Automorphism and noninvariant properites of the computably enumerable sets, $\mathrm{PhD}$ thesis, University of Chicago. 2.1

Department of Mathematics, University of Notre Dame, Notre Dame, IN 46556-5683

E-mail address: Peter. Cholak.1@nd.edu

Department of Mathematics, University of California, Berkeley, CA $94720-$ 3840

E-mail address: leo@math. berkeley.edu 